\title{
On the periodic solutions of the relativistic driven harmonic oscillator $^{1}$
}

\author{
Jaume Llibre ${ }^{1}$ and Amar Makhlouf ${ }^{2}$ \\ ${ }^{1}$ Departament de Matematiques, Universitat Autònoma de Barcelona, \\ 08193 Bellaterra, Barcelona, Catalonia, Spain \\ jllibre@mat.uab.cat \\ ${ }^{2}$ Department of Mathematics, Laboratory LMA, University of Annaba, \\ Elhadjar, 23 Annaba, Algeria \\ makhloufamar@yahoo.fr
}

ABSTRACT. Using the averaging theory we prove the existence of periodic orbits with small velocities with respect to the speed of light in the forced harmonic oscillator with relativistic effects in dimension one.

\section{Introduction AND StATEMENT of the MAin RESUlts}

For the forced harmonic oscillator with relativistic effects in dimension one with equation of motion

$$
\left(\frac{m x^{\prime}}{\sqrt{1-\frac{x^{\prime 2}}{c^{2}}}}\right)^{\prime}+k x=F_{0} \cos (\omega t),
$$

we prove the existence of periodic orbits with small velocities with respect to the speed of light. Here $c>0$ is the speed of light in the vacuum, $m>0$ is the mass at rest, $k>0$ is the spring stiffness coefficient, and $\omega$ and $F_{0}$ are the frequency and amplitude of the external force, see for more details on this equation [14].

These last years some results have been published on the periodic solutions of different oscillators with relativistic effects, as the relativistic forced pendulum, see for instance the works of Brezis and Mawhin [4], Bereanu and Torres [2], and Maró [13]. These authors have obtained their results using variational and topological methods. The stability of the equilibrium $x=0$ of the relativistic pendulum with variable length has been proved by Chu, Lei and Zhang in [8].

Kim and Lee in [10] have studied numerically the existence of chaotic motion in the relativistic harmonic oscillator. Moreover, the existence of chaotic motion of relativistic particles has been studied in different contexts by several authors, see for instance $[3,6,7,11]$.

\footnotetext{
${ }^{1} 2010$ Mathematics Subject Classification: Primary 34D20, 34C15. Key words: periodic solution, relativistic harmonic oscillator, averaging theory.
} 
Nuñez and Rivera in [14], following ideas of this previous work, and assuming that

(i) $\frac{k}{m}<\frac{\omega^{2}}{16}$,

(ii) $F_{0}<\frac{1}{4} m c \omega$, and

(iii) $\frac{(m c \omega)^{19}\left((m c \omega)^{2}-16 F_{0}^{2}\right)}{120 \pi F_{0}^{2}\left((m c \omega)^{2}+4 F_{0}^{2}\right)^{19 / 2}} \sin \left(\frac{6 \pi(\omega k)^{1 / 2} m c^{3 / 2}}{\left((m c \omega)^{2}+4 F_{0}^{2}\right)^{3 / 4}}\right)>1$,

proved the existence of a periodic orbit for the relativistic driven harmonic oscillator (1) via lower and upper solutions.

Our result shows the existence of periodic orbits in the relativistic driven harmonic oscillator with relativistic effects (1), under the assumptions that:

(i) the velocity of the periodic orbit is small with respect to the speed of light $c$, and

(ii) the quotient $\frac{\omega}{\sqrt{\frac{k}{m}}}=\frac{q}{p} \neq 1$, with $p$ and $q$ coprime positive integers.

Theorem 1. Under assumptions (i) and (ii) and for $\varepsilon>0$ sufficiently small the relativistic driven harmonic oscillator (1) has a periodic solution $x(t, \varepsilon)$ such that $\frac{x^{\prime}(t, \varepsilon)}{c}=O(\sqrt{\varepsilon})$ with period $2 p \pi \sqrt{\frac{m}{k}}$. Moreover $x(0, \varepsilon)=$ $\frac{F_{0}}{m \omega^{2}-1}+O(\varepsilon)=\frac{F_{0} p^{2}}{k\left(q^{2}-p^{2}\right)}+O(\varepsilon)$ and $x^{\prime}(0, \varepsilon)=O(\varepsilon)$ (see Figure 1).

Theorem 1 is proved in section 3 .

Note that the error in the initial conditions of the periodic solution of Theorem 1 is of order $\varepsilon$. Numerically it can be observed that for values of the velocity of the periodic solution less than 10 the approximation provided in Theorem 1 is useful.

The idea of the proof of Theorem 1 is the following. First we shall write the second-order differential equation (1) as a differential system of first order. Taking

$$
y=\frac{m x^{\prime}}{\sqrt{1-\frac{x^{\prime 2}}{c^{2}}}}
$$




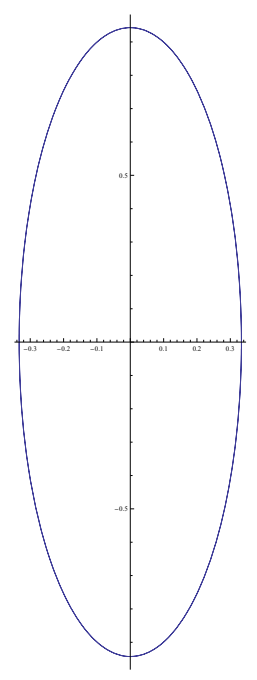

Figure 1. The periodic solution Theorem 1 for $m=2, c=$ $300000, F_{0}=1, k=1, p=1$ and $q=2$.

the differential equation of second order (1) can be written as the differential system of first order

$$
\begin{aligned}
& x^{\prime}=\frac{y}{\sqrt{m^{2}+\frac{y^{2}}{c^{2}}}}, \\
& y^{\prime}=-k x-F_{0} \cos (\omega t) .
\end{aligned}
$$

Therefore to find a periodic solution of the differential equation (1) is equivalent to find a periodic solution of the differential system (2).

Under our assumptions (i), i.e. the ratio of velocities $y / c$ is small, we consider the differential system (2) as a small perturbation of the differential system

$$
\begin{aligned}
x^{\prime} & =\frac{y}{m}, \\
y^{\prime} & =-k x-F_{0} \cos (\omega t) .
\end{aligned}
$$


The solution $\left(x\left(t ; x_{0}, y_{0}\right), y\left(t ; x_{0}, y_{0}\right)\right)$ of this system such that $\left(\left(x\left(0 ; x_{0}, y_{0}\right)\right.\right.$, $\left.y\left(0 ; x_{0}, y_{0}\right)\right)=\left(x_{0}, y_{0}\right)$ is

$$
\begin{aligned}
\left(x\left(t ; x_{0}, y_{0}\right)=\right. & \frac{1}{\sqrt{k m}\left(k-m \omega^{2}\right)}\left(\sqrt{k m}\left(F_{0}+x_{0}\left(k-m \omega^{2}\right)\right) \cos \frac{\sqrt{k} t}{m}\right. \\
& \left.-F_{0} \sqrt{k m} \cos (\omega t)+y_{0}\left(k-m \omega^{2}\right) \sin \frac{\sqrt{k} t}{m}\right), \\
y\left(t ; x_{0}, y_{0}\right)= & \frac{1}{k-m \omega^{2}}\left(y_{0}\left(k-m \omega^{2}\right) \cos \frac{\sqrt{k} t}{m}+m \omega F_{0} \sin (\omega t)\right) \\
& +\left(-\sqrt{k m}\left(F_{0}+k x_{0}-m x_{0} \omega^{2}\right) \sin \frac{\sqrt{k} t}{m}\right) .
\end{aligned}
$$

This periodic orbit has the period $2 \pi p \sqrt{\frac{m}{k}}$, recall that by assumption (ii) we have $\omega=\sqrt{\frac{k}{m}} \frac{q}{p}$.

In summary, all the solutions (4) of the differential system (3) are periodic with the same period $2 \pi p \sqrt{\frac{m}{k}}$. Since the relativistic driven harmonic oscillator $(2)$, when $y / c$ is small, can be considered a perturbation of the differential system (3), we shall study how many periodic solutions (4) of system (3) persist in system (2) when $y / c$ is sufficiently small. The tool for doing this study is the averaging theory, see section 2 for a summary of the results on the averaging theory that we shall need. Using the averaging theory we shall see that only one of the periodic orbits of system (3) persists for system (2). More precisely, the equality $x(0, \varepsilon)=\frac{F_{0}}{m \omega^{2}-1}+O(\varepsilon)=\frac{F_{0} p^{2}}{k\left(q^{2}-p^{2}\right)}+O(\varepsilon)$ which appears in the statement of Theorem 1, implies that the initial condition $x_{0}=x(0,0)$ of the periodic solution of the linear differential system (3) which can be continued to the relativistic driven harmonic oscillator (2) satisfies the condition $F_{0}+\left(k-m \omega^{2}\right) x_{0}=0$.

\section{Preliminary Results: Averaging theory}

We want to study the $T$-periodic solutions of the periodic differential systems of the form

$$
\mathbf{x}^{\prime}=F_{0}(t, \mathbf{x})+\varepsilon F_{1}(t, \mathbf{x})+\varepsilon^{2} F_{2}(t, \mathbf{x}, \varepsilon),
$$

with $\varepsilon>0$ sufficiently small, where $F_{0}, F_{1}: \mathbb{R} \times \Omega \rightarrow \mathbb{R}^{n}$ and $F_{2}: \mathbb{R} \times \Omega \times$ $\left(-\varepsilon_{0}, \varepsilon_{0}\right) \rightarrow \mathbb{R}^{n}$ are $\mathcal{C}^{2}$ functions, $T$-periodic in the variable $t$, and $\Omega$ is an open subset of $\mathbb{R}^{n}$. We denote by $\mathbf{x}(t, \mathbf{z}, \varepsilon)$ the solution of the differential system (5) such that $\mathbf{x}(0, \mathbf{z}, \varepsilon)=\mathbf{z}$. We assume that the unperturbed system

$$
\mathbf{x}^{\prime}=F_{0}(t, \mathbf{x}),
$$


has an open set $V$ with $\mathrm{Cl}(V) \subset \Omega$ such that for each $\mathbf{z} \in \mathrm{Cl}(V), \mathbf{x}(t, \mathbf{z}, 0)$ is $T$-periodic.

We consider the variational equation

$$
\mathbf{y}^{\prime}=D_{\mathbf{x}} F_{0}(t, \mathbf{x}(t, \mathbf{z}, 0)) \mathbf{y},
$$

of the unperturbed system on the periodic solution $\mathbf{x}(t, \mathbf{z}, 0)$, where $\mathbf{y}$ is an $n \times n$ matrix. Let $M_{\mathbf{z}}(t)$ be the fundamental matrix of the linear differential system (7) such that $M_{\mathbf{z}}(0)$ is the $n \times n$ identity matrix.

Theorem 2. Consider the function $\mathcal{F}: \mathrm{Cl}(V) \rightarrow \mathbb{R}^{n}$

$$
\mathcal{F}(\mathbf{z})=\int_{0}^{T} M_{\mathbf{z}}^{-1}(t) F_{1}(t, \mathbf{x}(t, \mathbf{z}, 0)) d t .
$$

If there exists $\alpha \in V$ with $\mathcal{F}(\alpha)=0$ and

$$
\operatorname{det}((d \mathcal{F} / d \mathbf{z})(\alpha)) \neq 0,
$$

then there exists a $T$-periodic solution $\mathbf{x}(t, \varepsilon)$ of system (5) such that $\mathbf{x}(0, \varepsilon)=$ $\alpha+O(\varepsilon)$.

The existence of the periodic solution of Theorem 2 is due to Malkin [12] and Roseau [15], for a shorter and easier proof see [5]. In Corollary 1 of [5], which corresponds to Theorem 2 instead of $\mathbf{x}(0, \varepsilon)=\alpha+O(\varepsilon)$ appears $\mathbf{x}(0, \varepsilon) \rightarrow \alpha$ when $\varepsilon \rightarrow 0$, but if you look at the proof of Theorem 3.1 one can verify that actually we have $\mathbf{x}(0, \varepsilon)=\alpha+O(\varepsilon)$. We note that Corollary 1 follows directly from Theorem 3.1.

\section{Proof of Theorem 1}

The differential system (2) is a Hamiltonian system with $H=\frac{k x^{2}}{2}+$ $c^{2} \sqrt{m^{2}+y^{2} / c^{2}}+F_{0} \cos (\omega t) x$. We assume that $\frac{|y|}{c}=O(\sqrt{\varepsilon})$, or equivalently $c=\frac{C}{\sqrt{\varepsilon}}$ with $\varepsilon>0$ small and $C$ a constant. That is, we assume roughly speaking that the velocity of the relativistic driven harmonic oscillator is very small with respect to the speed of the light. Then system (2) becomes

$$
\begin{aligned}
& x^{\prime}=\frac{y}{\sqrt{m^{2}+\frac{y^{2}}{c^{2}}}}=\frac{y}{m}-\varepsilon \frac{y^{3}}{2 C^{2} m^{3}}+O\left(\varepsilon^{2}\right), \\
& y^{\prime}=-k x-F_{0} \cos (\omega t) .
\end{aligned}
$$


This differential system is into the normal form (5) for applying the averaging theory to it, because using the notation of section 2 we have

$$
\begin{aligned}
& \mathbf{x}=(x, y), \\
& F_{0}=\left(\frac{y}{m},-k x-F_{0} \cos (\omega t)\right), \\
& F_{1}=\left(\frac{-y^{3}}{2 C^{2} m^{3}}, 0\right) .
\end{aligned}
$$

The variational differential system $\mathbf{y}^{\prime}=D_{\mathbf{x}} F_{0}(t, \mathbf{x}(t, \mathbf{z}, 0)) \mathbf{y}$, of the differential system $\mathbf{x}^{\prime}=F_{0}(t, \mathbf{x})$, i.e. of system (3), on every periodic orbit (4) is

$$
x^{\prime}=\frac{y}{m}, \quad y^{\prime}=-k x .
$$

The fundamental matrix $M(t)$ of this linear system (11) such that $M(0)$ is the identity matrix is

$$
M(t)=\left(\begin{array}{cc}
\cos \left(\sqrt{\frac{k}{m}} t\right) & \frac{1}{\sqrt{k m}} \sin \left(\sqrt{\frac{k}{m}} t\right) \\
-\sqrt{k m} \sin \left(\sqrt{\frac{k}{m}} t\right) & \cos \left(\sqrt{\frac{k}{m}} t\right)
\end{array}\right) .
$$

The averaged function $\mathcal{F}\left(x_{0}, y_{0}\right)=\left(\mathcal{F}_{1}\left(x_{0}, y_{0}\right), \mathcal{F}_{2}\left(x_{0}, y_{0}\right)\right)$ defined by

$$
\mathcal{F}(\mathbf{z})=\int_{0}^{T} M_{\mathbf{z}}^{-1}(t) F_{1}(t, \mathbf{x}(t, \mathbf{z}, 0)) d t,
$$

where $\mathbf{z}=\left(x_{0}, y_{0}\right)$ and $\mathbf{x}(t, \mathbf{z}, 0)=\left(x\left(t ; x_{0}, y_{0}\right), y\left(t ; x_{0}, y_{0}\right)\right)$ is the periodic solution given in (4), is

$$
\begin{aligned}
\mathcal{F}_{1}= & -\frac{3 y_{0}}{16 C^{2} k m^{3}\left(p^{2}-q^{2}\right)^{2}}\left(F_{0}^{2} m p^{2}\left(p^{2}+2 q^{2}\right)+\right. \\
& \left.2 F_{0} k m p^{2}\left(p^{2}-q^{2}\right) x_{0}+k\left(p^{2}-q^{2}\right)^{2}\left(k m x_{0}^{2}+y_{0}^{2}\right)\right), \\
\mathcal{F}_{2}= & \frac{3\left(F_{0} p^{2}+k\left(p^{2}-q^{2}\right) x_{0}\right)}{16 C^{2} k m^{2} 3\left(p^{2}-q^{2}\right)^{3}}\left(F_{0}^{2} m p^{2}\left(p^{2}+2 q^{2}\right)\right. \\
& \left.+2 F_{0} k m p^{2}\left(p^{2}-q^{2}\right) x_{0}+k\left(p^{2}-q^{2}\right)^{2}\left(k m x_{0}^{2}+y_{0}^{2}\right)\right) .
\end{aligned}
$$

The system

$$
\mathcal{F}_{1}\left(x_{0}, y_{0}\right)=0, \quad \mathcal{F}_{2}\left(x_{0}, y_{0}\right)=0,
$$

has the unique solution $\left(x_{0}, y_{0}\right)=\left(-\frac{F_{0} p^{2}}{k\left(p^{2}-q^{2}\right)}, 0\right)$, the other solutions either are complex, or their Jacobian (9) is zero. The Jacobian matrix of the 
function $\left(\mathcal{F}_{1}\left(x_{0}, y_{0}\right), \mathcal{F}_{2}\left(x_{0}, y_{0}\right)\right)$ at the solution $\left(x_{0}, y_{0}\right)=\left(-\frac{F_{0} p^{2}}{k\left(p^{2}-q^{2}\right)}, 0\right)$ is

$$
A=\left(\begin{array}{cc}
0 & -\frac{3 F_{0}^{2} p^{2} q^{2}}{8 C^{2} k m^{2}\left(p^{2}-q^{2}\right)^{2}} \\
\frac{3 F_{0}^{2} p^{2} q^{2}}{8 C^{2} m\left(p^{2}-q^{2}\right)^{2}} & 0
\end{array}\right) .
$$

The determinant of this matrix is $\operatorname{det}(A)=\frac{9 F_{0}^{4} p^{4} q^{4}}{64 C^{4} k m^{3}\left(p^{2}-q^{2}\right)^{4}}$, note that $p \neq q$ from the assumptions. By Theorem 2 we conclude that there is a periodic solution of system $(10)(x(t, \varepsilon), y(t, \varepsilon))$ such that $(x(0, \varepsilon), y(0, \varepsilon)) \rightarrow$ $\left(-\frac{F_{0} p^{2}}{k\left(p^{2}-q^{2}\right)}, 0\right)$ when $\varepsilon \mapsto 0$. So Theorem 1 is proved.

\section{ACKNowledgements}

The first author is partially supported by the Ministerio de Economía, Industria y Competitividad, Agencia Estatal de Investigación grants MTM201677278-P (FEDER) and MDM-2014-0445, the Agència de Gestió d'Ajuts Universitaris i de Recerca grant 2017SGR1617, and the H2020 European Research Council grant MSCA-RISE-2017-777911.

\section{REFERENCES}

[1] C. Bereanu and J. Mawhin, Existence and multiplicity results for some nonlinear problems with singular $\phi$-Laplacian, J. Differential Equations 243 (2007), 536-557.

[2] C. Bereanu and P.J. Torres, Existence of at least two periodic solutions of the forced relativistic pendulum, Proc. Amer. Math. Soc. 140 (2012), 2713-2719.

[3] M. Billardon, Storage ring free-electron laser and chaos, Physical Review Letters 65 (1990), 713-716.

[4] H. Brezis and J. Mawhin, Periodic solutions of the forced relativistic pendulum, Differential and Integral Equations 23 (2010), 801-810.

[5] A. Buica, J. P. Françoise and J. Llibre, Periodic solutions of nonlinear periodic differential systems with a small parameter, Comm. Pure Appl. Anal. 6 (2006), 103-111.

[6] C. Chen and R.C. Davidson, Chaotic particle dynamics in freeelectron lasers, Physical Review A 43 (1991), 5541-5554.

[7] A.A. Chernikov, T. Tél, G. Vattay and G. M. Zaslavsky, Chaos in the relativistic generalization of the standard map, Physical Review A 40 (1989), 4072-4076.

[8] J. Chu, J. Lei and M. Zhang, The stability of the equilibrium of a nonlinear planar system and application to the relativistic oscillator, J. Differential Equations 247 (2009), 530-542.

[9] D. Ionescu, Comparative analysis of the electrogravitational Kepler problem in GRT and RTG, Internat. J. Non-Linear Mech. 38 (2003), 1251-1268.

[10] J.H. Kim and H.W. Lee, Relativistic chaos in the driven harmonic oscillator, Physical Review E 51 (1995), 1579-1581.

[11] W.P. Leemans, C. Joshi, W.B. Mori, C.E. Clayton and T.W. Johnston, Nonlinear dynamics of driven relativistic electron plasma waves, Physical Review A 46 (1992), $5112-5122$. 
[12] I.G. Malkin, Some Problems of the theory of nonlinear oscillations, Gosudarstv. Izdat. Tehn-Teor. Lit. Moscow, 1956 (in Russian).

[13] S. Maró, Periodic solutions of a forced relativistic pendulum via twist dynamics, Topological Methods in Nonlinear Analysis 42 (2013), 51-75.

[14] D. Nuñez and A. Rivera, Twist periodic solutions in the relativistic driven harmonic oscillator, Abstract and Applied Analysis 2016, 6084082, 7 pp.

[15] M. Roseau, Vibrations non linéaires et théorie de la stabilité, Springer Tracts in Natural Philosophy, Vol. 8, Springer, New York, 1985. 